\title{
基于激发态分子内质子转移(ESIPT)原理的反应型荧光探针研究进展
}

\author{
王瑞祥 ${ }^{a}$ 赖晓静 ${ }^{a}$ 邱观音生*,b 刘晋彪 $*, a$ \\ ( ${ }^{a}$ 江西理工大学冶金与化学工程学院 赣州 341000) \\ $\left({ }^{b}\right.$ 嘉兴学院生物与化学工程学院 嘉兴 314001)
}

\begin{abstract}
摘要 基于激发态分子内质子转移(ESIPT)原理的反应型苂光探针, 因其具有高选择性、高灵敏度及大的斯托克斯位移 等优点而被广泛关注. 以检测目标物的属性归类，就近十年 ESIPT 反应型荧光探针进行综述，阐述其检测识别机制，并 对此类荧光探针应用中存在的问题及发展方向进行评述.

关键词 激发态分子内质子转移(ESIPT); 反应型; 苂光探针
\end{abstract}

\section{Recent Advances in Reaction-Based Excited State Intramolecular Proton Transfer (ESIPT) Fluorescence Probe}

\author{
Wang, Ruixiang $^{a} \quad$ Lai, Xiaojing $^{a} \quad$ Qiu, Guanyinsheng ${ }^{*, b} \quad$ Liu, Jinbiao ${ }^{*, a}$ \\ $\left({ }^{a}\right.$ School of Metallurgical and Chemical Engineering, Jiangxi University of Science and Technology, Ganzhou 341000) \\ ( ${ }^{b}$ College of Biological, Chemical Science and Engineering, Jiaxing University, Jiaxing 314001)
}

\begin{abstract}
Fluorescence detection based on excited state intramolecular proton transfer (ESIPT) using reaction-based probes has attracted considerable attention in the scientific community as they offers promising advantages, including high selectivity, high sensitivity and large Stoke shift. The representative examples of design strategies, mechanism of actions, existing challenges and future developments for reaction-based ESIPT fluorophores reported in the last ten years are reviewed.

Keywords excited state intramolecular proton transfer (ESIPT); reaction; fluorescence probe
\end{abstract}

荧光探针技术是借助探针分子与目标物结合，从而 改变探针分子的苂光性质, 最终实现对目标物质检测识 别的一种分析方法, 具有灵敏度高、检测限低、选择性 好和操作简便等优点 ${ }^{[1 \sim 4]}$. 苂光探针的设计原理有多种, 如光诱导电子转移 ${ }^{[5]}$ 、分子内电荷转移 ${ }^{[6,7]}$ 、荧光共振能 量转移 ${ }^{[811]}$ 、激发态分子内质子转移 (excited-state intramolecular proton transfer, ESIPT) ${ }^{[12]}$ 和聚集诱导发 光 ${ }^{[13,14]}$ 等. 其中, 基于 ESIPT 机理的苂光探针具有发光 强、斯托克斯位移较大、光稳定性好等优异特性, 表现 出良好的传感性能. 近来, 涌现出了大量有关 ESIPT 的 理论和实验研究.

一般来说, 如果分子的结构包含氢键供体 $(\mathrm{OH}$ 或 $\left.\mathrm{NH}_{2}\right)$ 和氢键受体 $(=\mathrm{N}$ 一和 $\mathrm{C}=\mathrm{O})$, 那么这类分子就能发 生分子内质子转移, 显示出 ESIPT 荧光. 最常见的具有 ESIPT 典型结构的化合物有 2-(2'-羟基苯基)苯并噁唑
(HBO)、2-(2'-羟基苯基)苯并噻唑(HBT)、2-(2'-羟基苯基) 苯并咪唑(HBI) 以及 3-羟基黄酮衍生物等(图 1). ESIPT 过程是苂光分子受光激发后, 激发态分子内部质子给体 与相邻的质子受体之间发生质子转移. 在激发态下质子 氢发生转移, 导致分子内共轭结构发生极大的变化, 光 谱发生红移，从而可以观察到一个很明显的 Stokes 位 移 ${ }^{[15]}$. 如图 2 所示, 以 HBT 为例, 烯醇式异构体 $\mathrm{E}$ (enol-form) 受到激发, 跃迁至单线激发态 $\mathrm{E}^{*}$, 随即快速 发生分子内质子转移, 得到酮式异构体单线激发态 $\mathrm{K}^{*}$, 最终以发射荧光的方式返回基态 $\mathrm{K}$ (keto-form). 相比 $\mathrm{E}$ 与 $\mathrm{E}^{*}$ 之间的能量间隙, $\mathrm{K}^{*}$ 与 $\mathrm{K}$ 之间的能量间隙更小. 由 此造成电子从激发态 $\mathrm{K} *$ 回落至基态 $\mathrm{K}$ 时发出的苂光波 长更长, 即展现出更大的 Stokes 位移.

反应型荧光探针是基于苂光分子与目标物质发生 化学反应，导致探针化学结构改变，从而使得探针的荧

\footnotetext{
* Corresponding authors. E-mail: liujbgood@hotmail.com; qiuguanyinsheng@mail.zjxu.edu.cn Received November 5, 2018; revised November 30, 2018; published online December 17, 2018 Project supported by the National Natural Science Foundation of China (Nos. 21762018, 21772067), the Natural Science Foundation of Jiangxi Province (No. 20171BAB213008) and the Program of Qingjiang Excellent Young Talents, Jiangxi University of Science and Technology.

国家自然科学基金(Nos. 21762018, 21772067)、江西省自然科学基金(No. 20171BAB213008)和江西理工大学清江青年英才计划资助项目
} 
<smiles>Oc1ccccc1-c1nc2ccccc2o1</smiles>

HBO<smiles>COc1ccc(C(=O)c2ccccc2)c(O)c1</smiles>

Benzophenone<smiles>Oc1ccccc1-c1nc2ccccc2s1</smiles>

HBT<smiles>Oc1ccccc1-c1nc2ccccc2[nH]1</smiles>

$\mathrm{HBI}$<smiles>O=c1c(O)c(Br)oc2ccccc12</smiles>

Flavone<smiles>Oc1ccccc1/C=N/c1ccccc1</smiles>

$N$-Salicylideneanlines<smiles>[R]NC(=O)c1nc2ccccc2[nH]c1=O</smiles><smiles>Oc1cccc2ccc3cccnc3c12</smiles>

Quinoline<smiles>Oc1ccccc1-n1nccn1</smiles>

Benzotriazole

图 1 具有 ESIPT 效应的代表性苂光团结构

Figure 1 Representative structures of commonly used ESIPT fluorophores
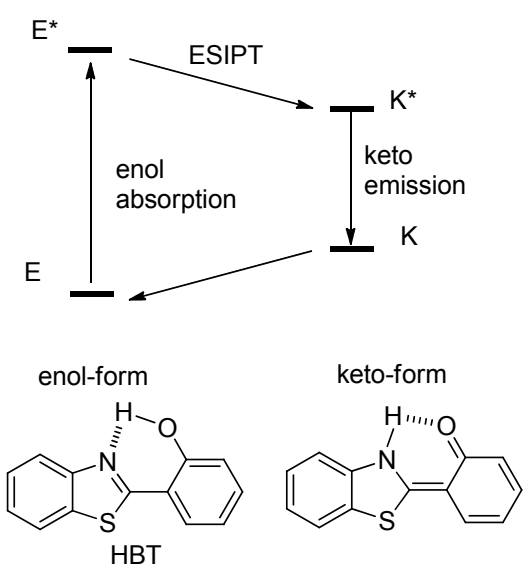

图 2 以 HBT 为例的 ESIPT 机理图

Figure 2 Mechanism of ESIPT using HBT as an example 光性质发生改变, 最终达到对目标物质检测的目的 ${ }^{[16]}$. 反应型荧光探针相较于传统荧光探针, 具有对目标物质 识别性更高、识别监测信息响应灵敏且时间较久等优点. 目前, 基于 ESIPT 机理设计的反应型苂光探针已成为研 究热点, 被广泛用于阴离子、金属离子、中性分子和生 物酶等物质的检测. 鉴于此, 本文将针对基于 ESIPT 机 理的反应型苂光探针研究进展进行总结和评述.

\section{1 阴离子荧光探针}

\section{$1.1 \mathrm{~F}^{-}$荧光探针}

氟离子在化学、生物、医学、环境和材料等领域都 有重要作用. 适量的氟对于人体有益, 但是过量的氟摄 入, 将导致氟骨症等多种疾病. 因此, 对于氟离子的检 测识别一直是分析化学家们关注的重点. 氟原子的电负 性较大, 并且 $\mathrm{F}^{-}$与硅具有极高的亲和力. Yang 等 ${ }^{[17]}$ 以 2-(2'-羟基苯基)-苯并咪唑作为底物, 与叔丁基二甲基氯 硅烷(TBS-Cl) 反应合成苂光探针 1 . 当溶液中存在 $\mathrm{F}^{-}$时, $\mathrm{F}^{-}$可以快速断开 $\mathrm{Si}-\mathrm{O}$ 键, 即使得探针 $\mathbf{1}$ 上的 $\mathrm{TBS}$ 基团 脱除, 从而恢复 ESIPT 结构, 展现出较大 Stokes 位移荧
光(Eq. 1). 基于 HBI 核心骨架的探针 $\mathbf{1}$, 能专一性地识 别 $\mathrm{F}^{-}$，操作方便，效果显著.

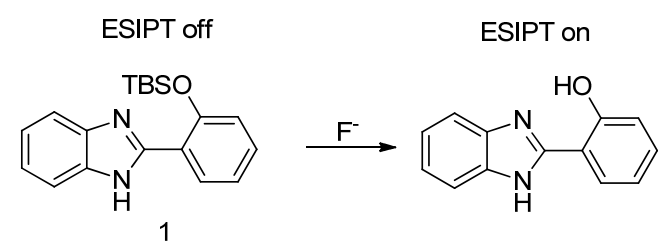

基于类似的思路，一系列氟离子苂光探针相继被开 发，例如基于喹啉骨架的探针 $\mathbf{2}^{[18]}$ 、HBT 骨架的探针 3 $5^{[19]}$ 、咪唑骨架的探针 $6^{[22]}$ (图 3). 由于氟离子对 $\mathrm{Si}-\mathrm{O}$ 键的选择性断开, 使得此类荧光探针具有很高的专一 性. 如表 1 所示, 基于 ESIPT 型苂光探针, 在脱除硅保 护基后，展现出较大的 Stokes 位移值. 此外，探针 $\mathbf{3}$ 和 $\mathbf{4}$ 还被应用于细胞成像，具有较好的应用前景.<smiles>Cc1ccc2c(Br)cc(Br)c(O[Si](C)(C)C(C)(C)C)c2n1</smiles>

2<smiles>CC(C)(C)[Si](Oc1ccc(NC(=O)c2ccccc2)cc1-c1nc2ccccc2s1)(c1ccccc1)c1ccccc1</smiles>

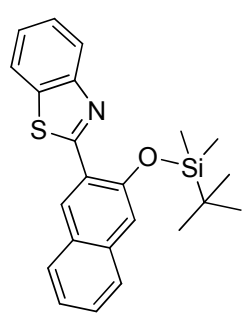

4<smiles>CC(C)(C)[Si](Oc1ccccc1-c1nc(-c2ccccc2)c(-c2ccccc2)[nH]1)(c1ccccc1)c1ccccc1</smiles>

图 3 探针 $2 \sim 6$ 的结构

Figure 3 Structures of probes $\mathbf{2} \sim \mathbf{6}$

由于 $\mathrm{F}^{-}$能够与活泼氢形成很稳定的氢键, 基于此, Saravanan 等 ${ }^{[23]}$ 设计合成了成苯并硒二唑基二芳基胺 $(7$, TBS-HN), 作为一种高选择性、高灵敏度的 $\mathrm{F}^{-}$苂光探针 
表 1 探针 1 6 相关检测性质比较

Table 1 Comparison of the detection properties of probes $1 \sim 6$

\begin{tabular}{cccc}
\hline Probe & $\lambda_{\text {ex }} / \mathrm{nm}$ & $\lambda_{\mathrm{em}} / \mathrm{nm}$ & Limit of detection \\
\hline $\mathbf{1}$ & 310 & 454 & $0.19 \mu \mathrm{mol} \cdot \mathrm{L}^{-1}$ \\
$\mathbf{2}$ & 335 & 520 & $1.0 \mu \mathrm{mol} \cdot \mathrm{L}^{-1}$ \\
$\mathbf{3}$ & 330 & 477 & $6.5 \mu \mathrm{mol} \cdot \mathrm{L}^{-1}$ \\
$\mathbf{4}$ & 360 & 586 & $10.18 \mu \mathrm{mol} \cdot \mathrm{L}^{-1}$ \\
$\mathbf{5}$ & 350 & 566 & $\approx 0.1 \mathrm{mg} / \mathrm{L}$ \\
$\mathbf{6}$ & 302 & 448 & $2.64 \mu \mathrm{mol} \cdot \mathrm{L}^{-1}$ \\
\hline
\end{tabular}

7. 当有 $\mathrm{F}^{-}$存在时，探针 7 拔除二芳胺上的氢，导致 ESIPT 过程将被抑制, 最终使得探针 7 的荧光性质发生 巨大变化, 实现对的 $\mathrm{F}^{-}$的选择性检测(Eq. 2). 有意思的 是, 在 $\mathrm{F}^{-}$存在下, 探针的颜色由红色转变为墨绿色, 肉 眼清晰可见，并且对于其它常见阴离子的选择性很高.

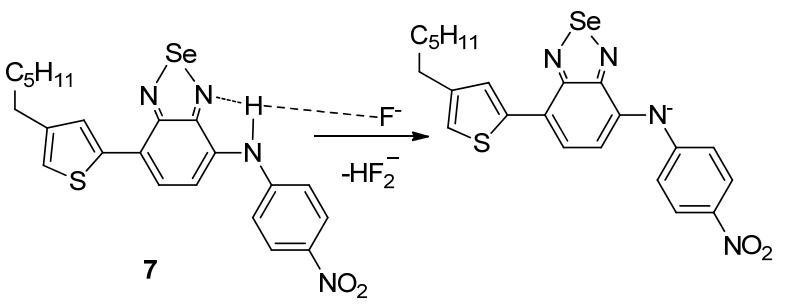

\section{$1.2 \mathrm{CN}^{-}$荣光探针}

$\mathrm{CN}^{-}$具有很强的亲核性能，能够对羰基或者亚胺进 行加成. Goswami 等 ${ }^{[24]}$ 应用 $\mathrm{CN}^{-}$的亲核性, 设计开发了 HBT 类苂光探针 8 , 该探针含有两个醛基, 能够与亲核 性的 $\mathrm{CN}^{-}$发生加成反应，改变探针的电子结构; 同时, 由于 $\mathrm{CN}^{-}$具有很强的氢键作用力，能拔除羟基上的氢， 进而抑制探针的 ESIPT 效应(Eq. 3). 通过以上两种作用, 探针的荧光发生明显变化, 苂光颜色从绿色转变成蓝 色. 该文作者通过核磁共振氢谱, 验证了 $\mathrm{CN}^{-}$确实与探 针发生了加成, 使得醛基氢转变为醇羟基氢.<smiles>CC(C)OC(C)c1cc(C=O)cc(-c2nc3ccccc3s2)c1O</smiles>

Jiang 等 ${ }^{[25]}$ 以 3,5-二叔丁基-2-羟基苯甲醛和 2-氨基 苯酚作为底物，通过简单的醛-胺缩合反应获得探针 9. 探针 9 在二甲亚砜(DMSO)溶液中不具荧光性, 加入 CN 一后，与亚胺发生亲核加成，随即发生亲核环化脱去 $\mathrm{CN}$ 一得到 $\mathrm{HBO}$ 类苂光产物(Scheme 1). $\mathrm{CN}^{-}$在该策略中起 到催化剂的作用, 得到具有 ESIPT 效应的苂光产物, 从 而实现了 $\mathrm{CN}^{-}$的识别. 该类荧光探针具有很好的专一 性，对于其它常见的阴离子都无响应.

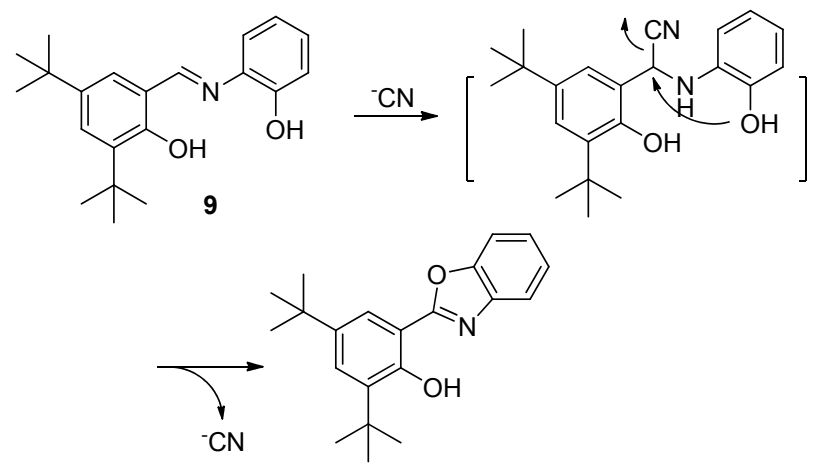

图式 1 探针 9 对 $\mathrm{CN}^{-}$的检测机理

Scheme 1 Reaction mechanism of probe 9 on the detection of $\mathrm{CN}^{-}$ion

\section{$1.3 \mathrm{SO}_{3}^{2-} / \mathrm{HSO}_{3}^{-}$荧光探针}

Yang 等 ${ }^{[26]}$ 以 2-(2'-羟基苯基)苯并噻唑作为原料, 合 成出探针 10. 探针 10 含有不饱和羰基结构, 当 $\mathrm{SO}_{3}^{2-}$ 存 在时, 不饱碳碳双键受到 $\mathrm{SO}_{3}^{2-}$ 的加成, 进而得到 $\mathrm{HBT}$ 类荧光产物，最终实现 $\mathrm{SO}_{3}^{2-}$ 的选择性识别(Eq. 4). 探针 10 的苂光可从橙色转变为蓝色, 并且被成功应用于活 体细胞中 $\mathrm{SO}_{3}^{2-}$ 的苂光检测.

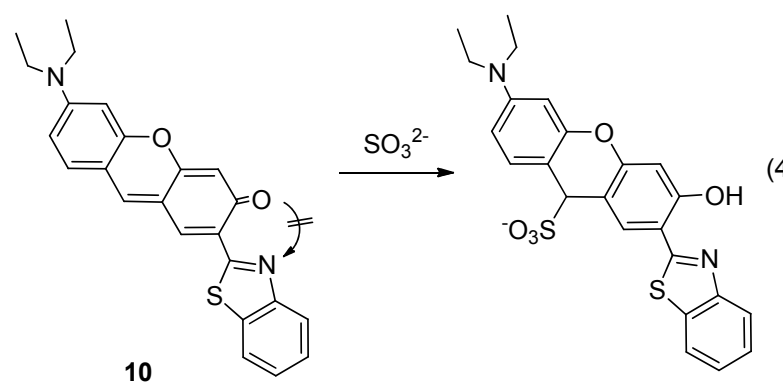

2016 年, Feng 等 ${ }^{[27]}$ 制备了一种新型的 HBT-半花菁 混合物荧光探针 11. 与传统的 ESIPT 染料 HBT 相比, 这 种杂化体具有更大的红移发射，可以用作比色和比率苂 光探针来检测亚硫酸氢盐. 该探针的检测机理为, $\mathrm{HSO}_{3}^{-}$对双键进行加成，破坏了整个大共轭结构，使得 苂光发射峰波长从 $610 \mathrm{~nm}$ 转变为 $485 \mathrm{~nm}(\mathrm{Eq} .5)$. 在水 溶液中具有高选择性和高灵敏度, 对 $\mathrm{HSO}_{3}^{-}$的检测极限 约 $56 \mathrm{nmol} \cdot \mathrm{L}^{-1}$, 线性范围为 $0 \sim 25 \mu \mathrm{mol} \cdot \mathrm{L}^{-1}$. 该探针的

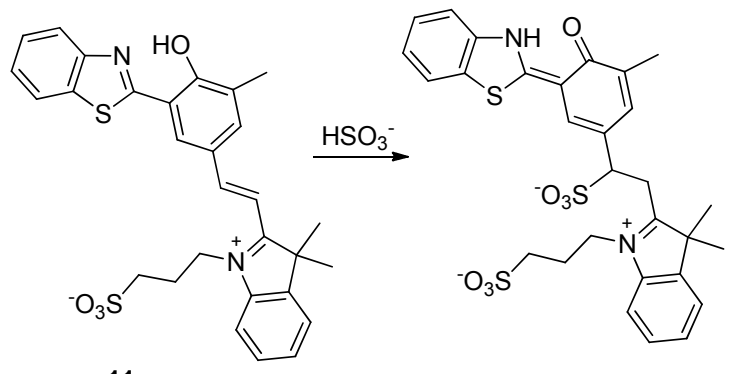


实际应用价值已通过对食物样品和活细胞中的 $\mathrm{HSO}_{3}^{-}$ 的检测试验结果得到证明.

\section{$1.4 \mathrm{~S}^{2-} / \mathrm{H}_{2} \mathrm{~S}$ 荧光探针}

Goswami 等 ${ }^{[28]}$ 为实现快速检测 $\mathrm{S}^{2-} / \mathrm{H}_{2} \mathrm{~S}$, 以 ESIPT 原理为基础, 设计合成了 $\mathrm{HBT}$ 类探针 12. 在 $\mathrm{S}^{2-} / \mathrm{H}_{2} \mathrm{~S}$ 存 在下, 探针 12 出现一个较大的红移发射. 该文作者指 出, 探针甲酰基首先受到 $\mathrm{S}^{2-}$ 的亲核进攻, 随后颈基与 酯基发生分子内酯交换, 得到典型的 HBT 荧光分子 (Scheme 2). 探针还被成功应用于酵母细胞的荧光成像. 仅由探针处理过的细胞无荧光, 在经过硫化钠和探针一 起处理过 $20 \mathrm{~min}$ 后, 细胞苂光成像明显.

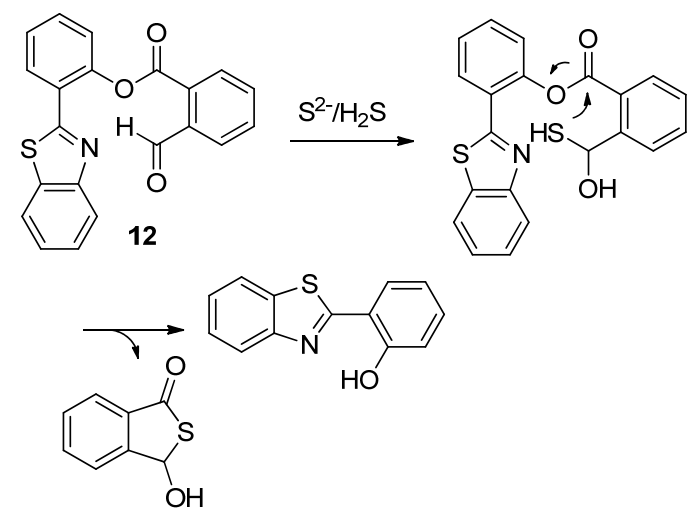

图式 2 探针 12 对 $\mathrm{S}^{2-}$ 的检测机理

Scheme 2 Reaction mechanism of probe 12 on the detection of $\mathrm{S}^{2-}$ ion

Yang 等 ${ }^{[29]}$ 基于 HBT 骨架的 ESIPT 原理, 开发了 $\mathrm{H}_{2} \mathrm{~S}$ 比率苂光探针 13. 该探针仅在 $374 \mathrm{~nm}$ 处显示烯醇 类苂光发射; 然而, 在中性溶液中引入 $\mathrm{H}_{2} \mathrm{~S}$ 后, 通过串 联亲核取代/环化反应除去探针的保护基团，使得探针 恢复 ESIPT 效应，导致 $374 \mathrm{~nm}$ 处的发射带减少，而 478 $\mathrm{nm}$ 处新的苂光峰不断增加(Scheme 3). 在 478 和 $374 \mathrm{~nm}$ 处的苂光强度比值, 随着 $\mathrm{H}_{2} \mathrm{~S}$ 浓度增加在 $0.5 \sim 10$

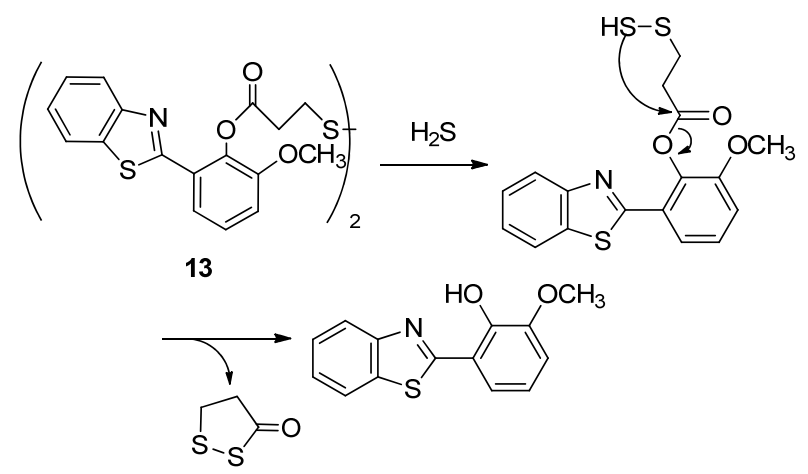

图式 3 探针 13 对 $\mathrm{H}_{2} \mathrm{~S}$ 的检测机理

Scheme 3 Reaction mechanism of probe $\mathbf{1 3}$ on the detection of $\mathrm{H}_{2} \mathrm{~S}$ 
蛋白(BSA)作用下，脱除保护基团，最终得到 HBT 型苂 光产物. 该荧光技术有助于更好地解释高碘酸盐相关的 生物和化学过程, 可以作为公共卫生、食品安全和环境 检测的有效工具.

\section{2 金属阳离子荧光探针}

\section{$2.1 \mathrm{Hg}^{2+}$ 荣光探针}

Doroshenko 等 ${ }^{[32]}$ 报道了一类基于 3 -羟基黄酮骨架 的苂光探针用于 $\mathrm{Hg}^{2+}$ 的检测, 但是有趣的是, 该检测机 理是通过 $\mathrm{Hg}^{2+}$ 与探针进行配位阻断 ESIPT 效应, 使得 荧光增强. 2011 年, Ahn 等 ${ }^{[33]}$ 基于 ESIPT 原理设计合成 了 HBT 骨架化合物 16, 作为 $\mathrm{Hg}^{2+}$ 的荧光探针(Eq. 6). 由于 $\mathrm{Hg}^{2}$ 能够促进烯基梄键的断裂, 进而获得 ESIPT 典型荧光结构; 并且相对其他金属离子, 选择性高. 往 探针 16 体系中加入 $\mathrm{Hg}^{2+}$, 探针的苂光发射峰从 $420 \mathrm{~nm}$ 漂移至 $500 \mathrm{~nm}$, 荧光显色灵敏. 该探针可在水溶液中进 行，对 $\mathrm{Hg}^{2+}$ 的检测限度可达 $20 \mathrm{ng} / \mathrm{L}$.

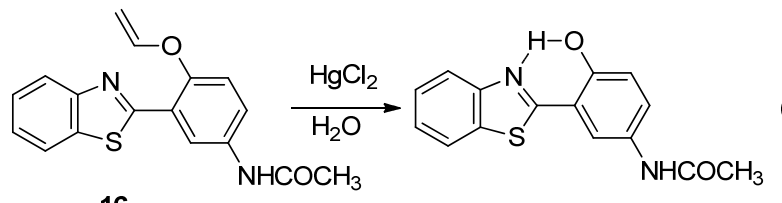

此外, $\mathrm{Hg}^{2+}$ 还能选择性脱除二硫代缩醛保护基. 基 于此机理, 2017 年 Chang 等 ${ }^{[34]}$ 设计合成了一类二噻烷衍 生物染料作为反应型 $\mathrm{Hg}^{2+}$ 苂光探针 17 (Eq. 7). 探针 17 在乙腈水溶液中对 $\mathrm{Hg}^{2+}$ 具有明显的苂光信号, 并且以 柠檬酸盐作为掩蔽剂和缓冲剂, 能够有效地去除 $\mathrm{Cu}^{2+}$ 离子的干扰响应. 该响应过程可在 $15 \mathrm{~min}$ 内完成, 并且 检测限可达 $2.0 \times 10^{-7} \mathrm{~mol} \cdot \mathrm{L}^{-1}$.

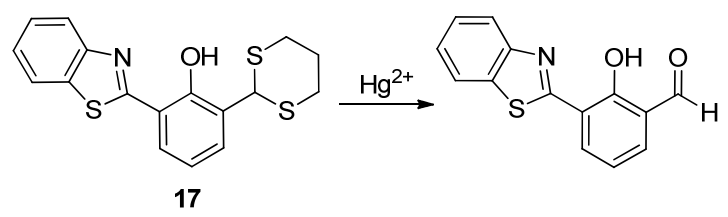

\section{$2.2 \mathrm{Cu}^{2+}$ 苂光探针}

通常, $\mathrm{Cu}^{2+}$ 能够使得苂光团发生苂光淬灭. 2015 年, Tang 等 ${ }^{[35]}$ 报道了一类 3-羟基黄酮类 “关-开-关” 型苂 光探针, 可以实现铝离子和铜离子的串联检测. 当铝离 子与探针发生络合时, 苂光得到增强; 随后加入铜离子, 荧光淬灭, 从而实现了两种离子的串联检测. 同年, Han 等 ${ }^{[36]}$ 报道了一种基于 ESIPT 机制的新型反应型 $\mathrm{Cu}^{2+}$ 苂 光探针 18. 该探针含有 2-吡啶甲酸酯, 能够选择性地被 $\mathrm{Cu}^{2+}$ 促进酯水解过程的发生, 从而使得 HBT 的 ESIPT 效应得到释放(Eq. 8). 该探针被成功应用于 HeLa 细胞
中: 当 $\mathrm{HeLa}$ 与探针 $\left(5 \mu \mathrm{mol} \cdot \mathrm{L}^{-1}\right)$ 捊化 $0.5 \mathrm{~h}$ 后, 加入氯化 铜 $\left(15 \mu \mathrm{mol} \cdot \mathrm{L}^{-1}\right)$ 溶液, 细胞苂光明显增强, 展现出较好 的应用前景.

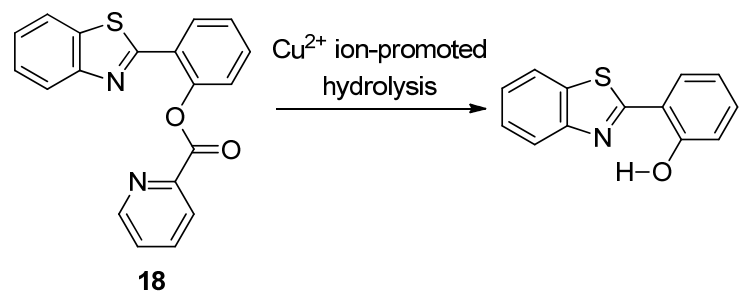

\section{$2.3 \mathrm{Pd}^{2+}$ 荧光探针}

相比烯基梄键，炔丙基或烯丙基醚键可被 $\mathrm{Pd}^{2+}$ 选择 性断开. 2012 年, Bai 等 ${ }^{\left[{ }^{37]}\right.}$ 设计了一类基于 3-差基黄酮骨 架的苂光探针 19. 该探针 3-位羟基通过炔丙基修饰，导 致 ESIPT 效应受阻; 在加入 $\mathrm{Pd}^{2+}$ 后, $\mathrm{Pd}^{2+}$ 选择性地断开 炔丙基醚键，使得探针恢复 ESIPT (Eq. 9). 该探针可以 实现各种氧化态钯的检测，检测限可达 $87 \mathrm{nmol} \cdot \mathrm{L}^{-1}$. 类 似地利用丙烯基与 $\mathrm{Pd}^{2+}$ 选择性反应，可脱除烯丙基，使 得探针恢复 ESIPT 结构, 苂光增强. Xu 等 ${ }^{[38]}$ 设计了一类 $\mathrm{HBT}$ 骨架化合物作为 $\mathrm{Pd}^{2+}$ 反应型苂光探针. 由于 $\mathrm{Pd}^{2+}$ 作为 Suzuki 反应的催化剂, 被广泛应用于药物分子的合 成中. 因此, $\mathrm{Pd}^{2+}$ 在药物分子中的残留检测，非常有必 要. 通过将该探针制成试纸, 对 Suzuki 反应产物进行检 测, 发现经过一次柱层析后的产物仍然有 $\mathrm{Pd}^{2+}$ 的残留. 当进行三次纯化后, 产物中的 $\mathrm{Pd}^{2+}$ 可降低到 $10 \mathrm{mg} / \mathrm{L}$ 以 下.

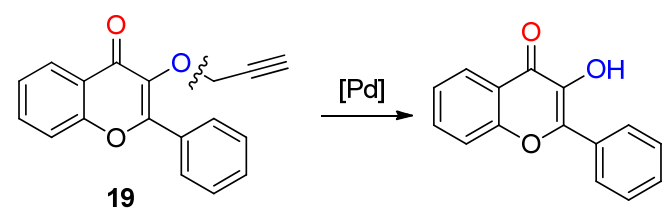

2017 年, Liu 等 ${ }^{[39]}$ 基于 3-着基黄酮骨架设计合成了 探针 20. 与探针 19 不同的是, 其保护基为烯丙氧羰基, 能够与钯进行选择性反应. 并且, 该探针可用于检测所 有典型氧化态 $(0,+2,+4)$ 的钯物种(Eq. 10). 在室温 下，探针 20 在大约 $1.5 \mathrm{~min}$ 内显示对钯物质的比率苂光 响应，具有较低的检测限 $\left(9.0 \mathrm{nmol} \cdot \mathrm{L}^{-1}\right)$. 与其他报道的 钯探针相比，具有检测条件温和、检测耗时短和较低的 检测限等优点.<smiles>C=CCOC(=O)c1c(-c2ccc(N(C)C)cc2)oc2ccccc2c1=O</smiles> 


\section{4 其他金属离子荧光探针}

除了发生共价键的断裂, 金属离子可与探针进行络 合, 阻碍探针的 ESIPT 效应的同时, 苂光得到增强. 此 类探针通常有较大的斯托克斯位移, 但是当 ESIPT 效应 发生阻碍时, 斯托克斯位移减小, 发射峰明显蓝移. 例 如, Zhang 等 ${ }^{[00]}$ 报道了一类水溶性嘧啶并苯并咪唑的苂 光探针 21 , 用于 $\mathrm{Al}^{3+}$ 的可视化检测. 研究发现, 在检测 体系中加入 $\mathrm{Al}^{3+}$ 后, 苂光光谱强度增强约 20 倍, 激发峰 由 $350 \mathrm{~nm}$ 红移到 $405 \mathrm{~nm}$, 发射峰由 $520 \mathrm{~nm}$ 蓝移到 505 nm. Gupta 等 ${ }^{[41]}$ 也报道了一例 $\mathrm{Zn}^{2+}$ 苂光探针 22, 机理也 是通过离子络合, 使得荧光团的 ESIPT 发生阻断, 但是 发生络合后, 苂光团的刚性结构使得荧光大大增加, 并 出现发射峰的明显蓝移.<smiles></smiles><smiles></smiles>

\section{3 氧化物荧光探针}

活性氧(ROS)在神经退行性疾病中起着重要作用, 同时在生物系统和自然界中也广泛存在. 基于 ROS 在 健康和环境中扮演的重要角色, ROS 的检测吸引了很多 关注. 2013 年, Churchill 等 ${ }^{[42]}$ 基于 ESIPT 原理设计合成 了两种反应型荧光探针 23 和 24 , 实现了对超氧化物 $\mathrm{KO}_{2}$ 的苂光检测(Eq. 11). 在超氧根离子存在下, 探针中 的醚键发生水解, 使得 HBT 结构恢复, 苂光大大增强. 两类探针选择性均很好, 并且观察到苂光红移达 $85 \mathrm{~nm}$, 荧光强度增大约 60 倍, 对超氧根离子的检测限可达 $6.38 \times 10^{-5} \mathrm{~mol} \cdot \mathrm{L}^{-1}$.

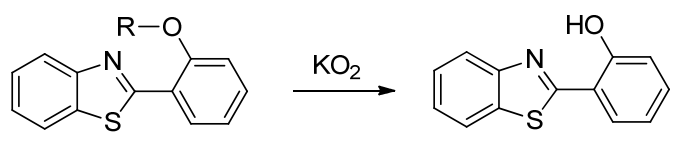

\footnotetext{
23: $\mathrm{R}=\mathrm{Ph}_{2} \mathrm{OP}$

24: $\mathrm{R}=4-\mathrm{O}_{2} \mathrm{NC}_{6} \mathrm{H}_{4}$
}

Xue 等 ${ }^{[43]}$ 基于 ESIPT 原理, 以 $\mathrm{HBO}$ 作为苂光团设 计合成了含喼酸酯识别基团的 $\mathrm{H}_{2} \mathrm{O}_{2}$ 苂光探针 25 (Eq. 12). HBO 类似物是典型的苂光化合物, 表现出独特的 聚集诱导发射增强现象. 此外, 探针含包含一长脂肪链, 目的是在含有表面活性剂体系下, 发生共聚, 在获得 ESIPT 效应的同时, 还观测到明显的聚集诱导发光. 在 双氧水存在下, 嗍酸酯茮基保护基团被氧化离去, 得到 $\mathrm{HBO}$ 骨架, 荧光最大发射峰从 $405 \mathrm{~nm}$ 移动到 $510 \mathrm{~nm}$,
红移明显.

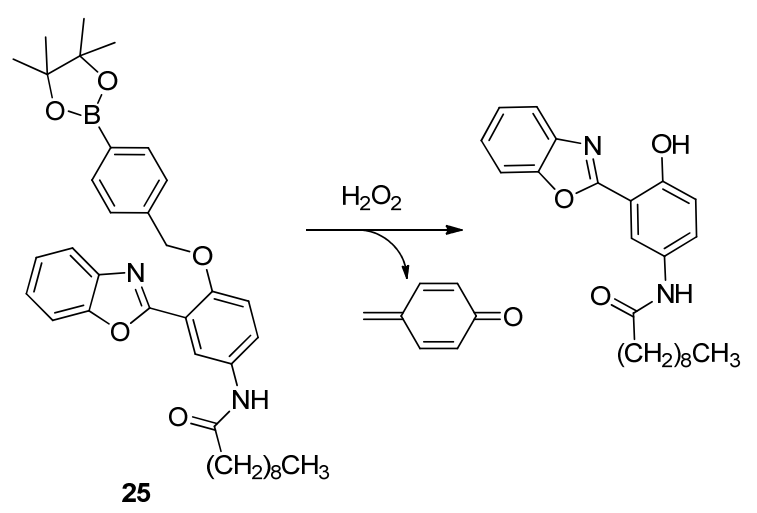

利用类似的机理, Liu 等 ${ }^{[4]}$ 设计合成了基于 $\mathrm{HBT}$ 骨 架的探针 26, 用于苂光检测过氧化苯甲酰. 虽然双氧水 也能氧化硼酸酯, 但是该探针能够选择性地区分双氧水 和过氧苯甲酰. 推测可能因为硼酸酯的位阻效应, 以及 检测体系中的乙醇降低了双氧化水的氧化性. 该探针还 被应用于 HeLa 细胞中过氧苯甲酰的苂光检测.

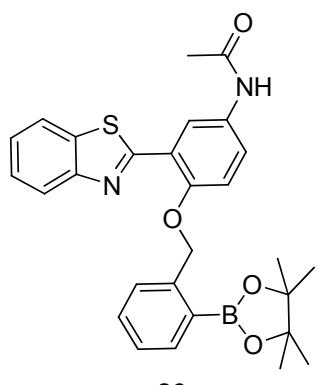

26

\section{4 生物小分子及酶荧光探针}

\section{1 半胱氨酸探针}

氨基酸小分子以及生物酶在生命过程中起到非常 关键的作用, 因此通过荧光手段对这些物质进行检测, 进而了解生命过程是非常有意义的. 2014 年 Pang 等 ${ }^{[45]}$ 报告了一种简单易用的荧光探针 27 , 用于生物学重要 的半胱氨酸(Cys)的快速特异性检测. 该探针以在可见 光下可发生 ESIPT 的染料 3-羟基黄酮作为荧光团, 并以 一个丙烯酸酯基团作为 ESIPT 阻断剂以及识别单元. 丙 烯酸酯部分可以在温和条件下与 Cys 在水溶液中发生特 异性反应，且快速地实现裂解，从而导致探针 27 恢复 ESIPT 过程(Eq. 13). 重要的是, 该探针能够将 Cys、高 半胱氨酸以及谷胱甘肽进行有效区分. 对 Cys 的检测限 为 $1 \mu \mathrm{mol} \cdot \mathrm{L}^{-1}$, 在活细胞中被成功应用于细胞内 $\mathrm{Cys}$ 的 生物成像, 表明该探针 27 具有很大的生物学应用潜力. 
<smiles>C=CC(=O)Oc1c(-c2ccccc2)oc2ccccc2c1=O</smiles>

基于类似检测机理, Feng 等 ${ }^{[46]}$ 也设计合成了 3-羟基 黄酮骨架探针 28, 用于特异性检测 Cys. Li 等 ${ }^{[47]}$ 以 3-(4,5-二苯基-1 $H$-咪唑-2-基)菜-2-酚为 ESIPT 荧光团, 设计合成了 Cys 探针 29, 同样带有丙烯酸酯识别基团.

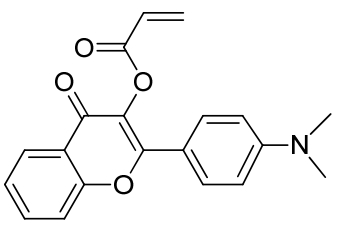

28

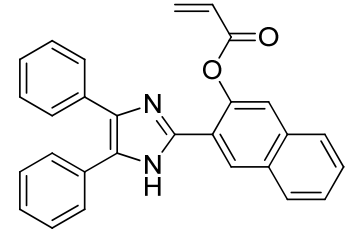

29
此外，甲酰基也被用作 Cys 的识别基团. Goswami 等 ${ }^{[48]}$ 以 $\mathrm{HBI}$ 为骨架设计合成了探针 $\mathbf{3 0}$, 用以检测水以 及细胞中的 Cys 和高半胱氨酸. 其反应机理以检测 Cys 为例, 主要是羰基氧与羟基氢能形成氢键, 从而阻碍了 ESIPT 效应; 在 Cys 或高半胱氨酸与甲酰基缩合成噻唑 环后, ESIPT 效应得到恢复(Eq. 14). 探针苂光发射峰从 $436 \mathrm{~nm}$ 红移到 $521 \mathrm{~nm}$.

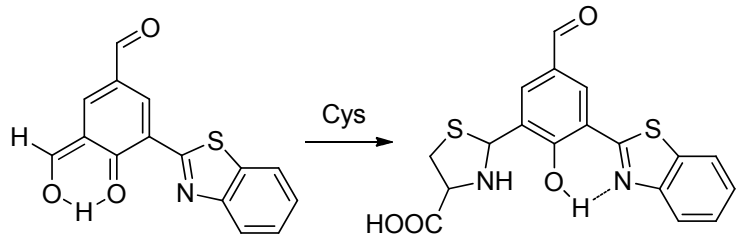

30

\section{2 磷酸酯酶探针}

酪氨酸磷酸酶(PTP)在细胞调节和信号转导通路中 起着关键作用, 包括癌症、糖尿病和阿尔茨海默氏症在 内的一些疾病都与磷酸酶的异常活性有关, 因此, PTP 的监测在生物医学研究中具有重要意义. Kim 等 ${ }^{[49]}$ 以 HBT 作为底物, 设计合成了 PTP 苂光探针 31. 该苂光探 针含有磷酸酯键, 能够与磷酸酶 MKP-6 特异性结合, 并 使探针上磷酸基团脱去恢复 ESIPT 结构, 最终实现 MKP-6 的苂光检测(Eq. 15). 研究发现, 在 26 种人酪氨 酸磷酸酶中, 仅有 MKP-6 能够实现该探针的磷酸酯基 水解, 选择性高.

类似的, Fan 等 ${ }^{[50]}$ 以 $\mathrm{HBI}$ 为骨架, 设计合成了含有 磷酸基团的苂光探针 32, 用以检测碱性磷酸酶(ALP).

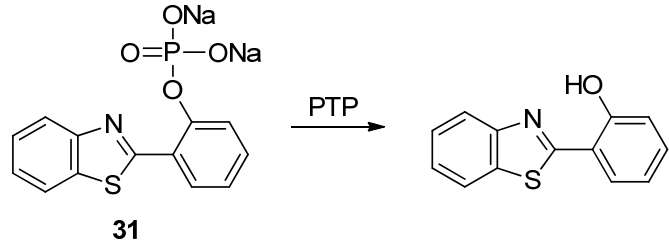

该探针对于 ALP 的检出限为 1.3 U/L. 2015 年, Zeng 等 ${ }^{[51]}$ 也报道了以 3-羟基黄酮为核心骨架的探针 33, 用以检 测 ALP, 检出限可达 $0.032 \mathrm{U} / \mathrm{L}$. 对于在生物实验中测定 ALP 水平是足够有效的，因为对于健康的成年人来说， 血液中的 ALP 水平在 $46 \sim 190 \mathrm{U} / \mathrm{L}$ 之间.

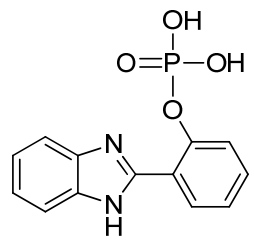

32<smiles>Cc1ccc(-c2oc3ccccc3c(=O)c2OP(=O)(O)O)cc1</smiles>

33
溶酶体酶负责细胞内各种蛋白质、脂质和碳水化合 物的消化, 其功能失调会导致许多遗传性溶酶体存储障 碍. 例如，溶酶体酯酶的失效会导致沃尔曼病，其症状 包括腹泻、腹部肿胀、肝脏肿大、体重无法增加等. Liu 等 ${ }^{[52]}$ 基于 ESIPT 原理构建了溶酶体酯酶苂光探针 34 . 该探针基于水杨酰肼荧光基团，其与酯酶反应，乙酰基 脱除, ESIPT 效应恢复，苂光大幅度增强(Eq. 16). 该探 针具有 AIE 和 ESIPT 的特点, 具有溶酶体特异性靶向、 高浓度不自淬灭、大斯托克斯移位、低细胞毒性和对酯 酶的高度特异性. 它也被用于原位监测溶酶体酯酶活性 和跟踪活细胞中的溶酶体运动, 这对于由溶酶体酯酶缺 乏引起的相关疾病的诊断具有很大的潜力.

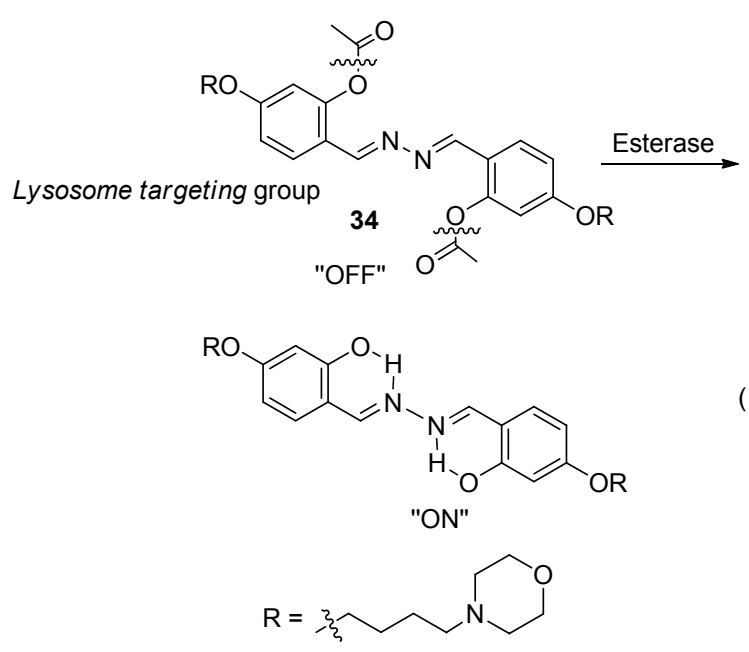

\section{5 其他分子荧光探针}

一氧化氮(NO)存在于心血管系统、免疫系统和神经 
系统中, 在生理和病理过程中都起着至关重要的作用. 许多疾病与体内 NO 的失调有关, 如癌症、缺血、感染 性休克、炎症、神经变性等. Zhu 等 ${ }^{[53]}$ 设计合成了用于 检测硝酰基 $(H N O)$ 的 ESIPT 型荧光探针 35. 该探针含有 3-羊基黄酮荧光团, 并通过酯基相连的识别受体一二 苯基膦苯甲酰基, 其与 $\mathrm{HNO}$ 反应形成氮杂叶立德, 随 后氮杂叶立德与羰基发生分子内亲核环化, 导致苂光团 3-羊基黄酮的释放(Eq. 17). 结果表明, 探针 35 对 HNO 的选择性响应优于其他生物还原剂, 能在 $0 \sim 20 \mu \mathrm{mol}$ $\mathrm{L}^{-1}$ 范围内定量检测 $\mathrm{HNO}$, 水溶液检测限为 $1.28 \times 10^{-7}$ $\mathrm{mol} \cdot \mathrm{L}^{-1}$. 该探针被成功应用于复杂生物样品(血清)2 $20 \mu \mathrm{mol} \cdot \mathrm{L}^{-1}$ 范围内的 $\mathrm{HNO}$ 检测, 其检测限为 $3.96 \times$ $10^{-7} \mathrm{~mol} \cdot \mathrm{L}^{-1}$, 具有较高实际应用价值.

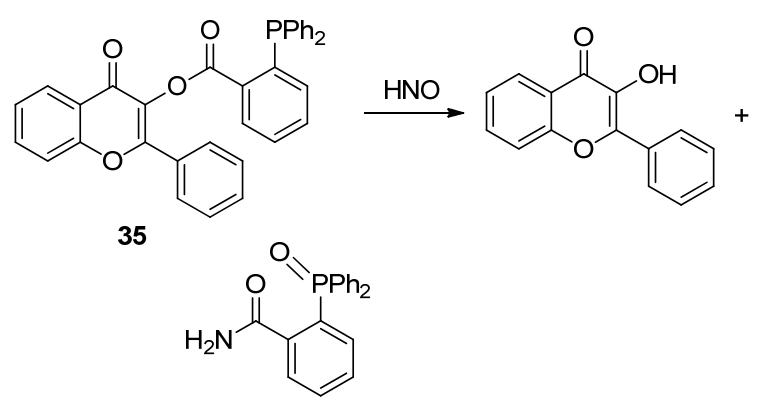

肼在化工、医药、农业等行业中常用作催化剂、缓 蚀剂、纺织染料、医药中间体等. 肼具有高毒性, 吸入 后可影响人和动物的肺、肝、脾、甲状腺等. Goswami 等 ${ }^{[54]}$ 基于 ESIPT 原理构建肼荧光探针 36, 其羟基被 $\gamma$ 溴代丁酰基保护, 从而导致 HBT 部分的 ESIPT 阻断. 当 加入肼时, 肼取代溴原子, 随后与酯基环化脱除保护基, 从而恢复 ESIPT, 实现肼的荧光检测(Eq. 18). 基于类似 的机理, Zhu 等 ${ }^{[55]}$ 设计合成了基于 3-羟基黄酮骨架的肼 荧光探针 37, 并将其用于在 HeLa 细胞中肼的检测.

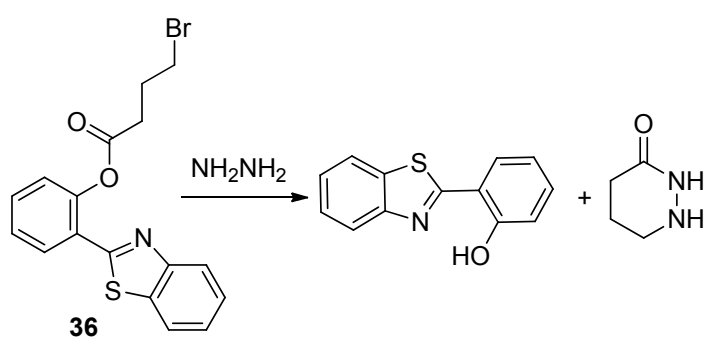<smiles>O=C(CCBr)Oc1c(-c2ccccc2)oc2ccccc2c1=O</smiles>

2014 年, Pang 等 ${ }^{[56]}$ 设计合成了一类3-乙酰氧基黄酮
类苂光探针 38 用于肼的检测. 他们通过对羟基进行酯 化修饰，阻碍荧光团的 ESIPT 效应; 在肼存在下, 肼能 够使得酯键发生断裂，从而使得 ESIPT 效应恢复，苂光 大大增强(Eq. 19). 探针具有优良的选择性, 可以区分其 他含氮试剂, 如三乙胺、羟胺、尿素、吡咯烷等. 并且 该探针还被应用到斑马鱼中的活体成像，表现出活体分 析检测肼的潜力. 最近, $\mathrm{Ju}$ 等 ${ }^{[57]}$ 也报道了一例基于 3 -羟 基黄酮骨架的用于检测肼的荧光探针 39. 其中，修饰基 团上 2 位的氯和 4 位的硝基均为拉电子基团, 有利于肼 解反应发生. 探针能够应用于活细胞中肼的微量检测, 展现其在生物活体分析方面潜在的应用价值.

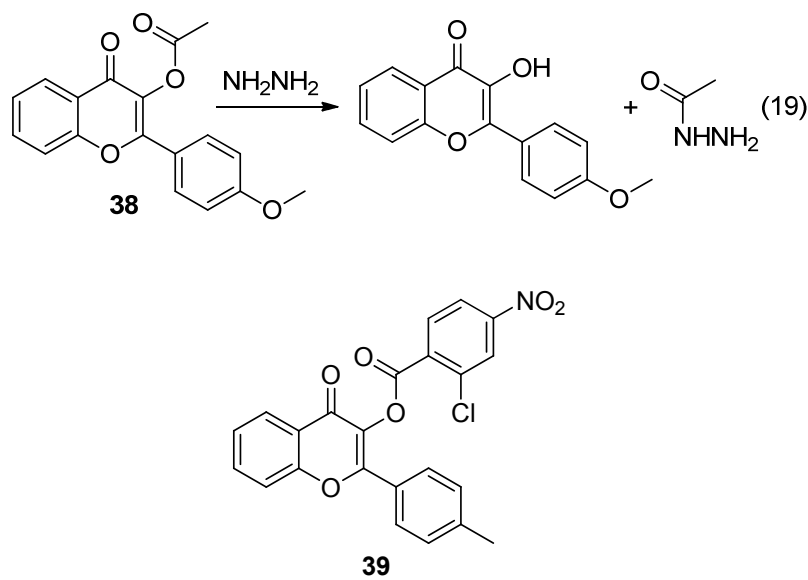

\section{6 结论与展望}

综上可见，基于 ESIPT 原理的反应型苂光探针，由 于其检测灵敏、选择性好、荧光发射红移明显以及 Stokes 位移大等优点, 已经得到很大发展. 然而现有的 ESIPT 反应型荧光探针仍存在诸多不足: (1)目前开发的 ESIPT 反应型荧光探针，发射波长谱段主要为 $400 \sim 600 \mathrm{~nm}$; 而较短的发射波长导致荧光在生命体内穿透性较弱, 不 利于在生物体内进行监测. (2)在质子性溶剂中的 ESIPT 的过程易受溶质一溶剂分子间氢键影响，其在水溶液中 质子转移能力比较弱, 因此不利于 ESIPT 反应型苂光探 针在活体细胞内的检测. 开发具有灵敏度高、选择性高、 光稳定性高、细胞膜通透性好等性能的 ESIPT 反应型苂 光探针, 尤其是一些生物酶的检测以及在疾病诊断中的 应用, 将成为研究热点.

\section{References}

[1] Zhang, H.-M.; Wu, Y.-C.; You, J.-Y.; Cao, L.; Ding, C.; Jiang, K.; Wang, Z.-Y. Chin. J. Org. Chem. 2016, 36, 2559 (in Chinese). (张惠敏, 吴彦城, 尤嘉宜, 曹梁, 丁沙, 蒋凯, 汪朝阳, 有机化 学, 2016, 36, 2559.)

[2] Xu, Q.-C.; Jin, C.; Zhu, X.-H.; Xing, G.-W. Chin. J. Org. Chem. 2014, 34, 647 (in Chinese). (徐勤超, 金灿, 朱雪慧, 邢国文, 有机化学, 2014, 34, 647.)

[3] Li, Y.-J.; Lv, Z.-Q.; Liu, M.; Xing, G.-W. Chin. J. Org. Chem. 2016, 
36, 962 (in Chinese).

(李杨洁, 吕子奇, 刘敏, 邢国文, 有机化学, 2016, 36, 962.)

[4] Zhang, C.; Gao, Y.-L. Chin. Ind. Eng. Prog. 2016, 35, 3288 (in Chinese).

(张聪，高云玲，化工进展, 2016, 35, 3288.)

[5] Ooyama, Y.; Matsugasako, A.; Oka, K.; Nagano, T.; Sumomogi, M.; Komaguchi, K.; Image, I.; Harima, Y. Chem. Commun. 2011, 47, 4448.

[6] Liu, Y.; Han, M.; Zhang, H.-Y.; Yang, L.-X.; Jiang, W. Org. Lett. 2008, 10, 2873

[7] Bryce, M. R. Adv. Mater. 2010, 11, 11 .

[8] Medintz, I. L.; Clapp, A. R.; Brunel, F. M.; Tiefenbrunn, T.; Uyeda, H. T.; Chang, E. L.; Deschamps, J. R.; Dawson, P. E.; Mattoussi, H. Nat. Mater. 2006, 5, 581.

[9] Marras, S. A.; Kramer, F. R.; Tyagi, S. Nucleic Acids Res. 2002, 30, e122.

[10] Clapp, A. R.; Medintz, I. L.; Mauro, J. M.; Fisher, B. R.; Bawendi, M. G.; Mattoussi, H. J. Am. Chem. Soc. 2004, 126, 301

[11] Berezin, M. Y.; Achilefu. S. Chem. Rev. 2010, 110, 2641.

[12] Kwon, J. E.; Park, S. Y. Adv. Mater. 2011, 23, 3615.

[13] Hong, Y.; Lam, J. W.; Tang, B. Z. Chem. Soc. Rev. 2011, 40, 5361.

[14] Mei, J.; Hong, Y.; Lam, J. W.; Qin, A.; Tang, Y.; Tang, B. Z. Adv. Mater. 2014, 26, 5429.

[15] Jiang, K.; Cao, L.; Hao, Z.-F.; Chen, M.-Y.; Chen, J.-R.; Li, X.; Xiao, P.; Chen, L.; Wang, Z.-Y. Chin. J. Org. Chem. 2017, 37, 2221 (in Chinese). (蒋凯, 曹梁, 郝志峰, 陈美燕, 程洁銮, 李晓, 肖萍, 陈亮, 汪 朝阳，有机化学, 2017, 37, 2221.)

[16] Cho, D. G.; Sessler, J. L. Chem. Soc. Rev. 2009, 38, 1647.

[17] Yang, X.-F.; Qi, H.; Wang, L.; Su, Z.; Wang, G. Talanta 2009, 80, 92.

[18] Bao, Y.; Liu, B.; Wang, H.; Tian, J.; Bai, R. Chem. Commun. 2011, 47, 3957.

[19] Goswami, S.; Das, A. K.; Manna, A.; Maity, A. K.; Fun, H. K.; Quah, C. K.; Saha, P. Tetrahedron Lett. 2014, 55, 2633.

[20] Mahapatra, A. K.; Mondal, S.; Manna, S. K.; Maiti, K.; Maji, R.; Ali, S. S.; Mandal, D.; Uddin, M. R.; Mandal, S. Supramol. Chem. 2016, 28, 693.

[21] Hu, R.; Feng, J.; Hu, D.; Wang, S.; Li, S.; Li, Y.; Yang, G. Angew. Chem., Int. Ed. 2010, 122. 5035.

[22] Dhanunjayarao, K.; Mukundam, V.; Venkatasubbaiah, K. Sens. Actuators, B 2016, 232, 175.

[23] Saravanan, C.; Easwaramoorthi, S.; Hsiow, C. Y.; Wang, K.; Hayashi, M.; Wang, L. Org. Lett. 2014, 16, 354.

[24] Goswami, S.; Manna, A.; Paul, S.; Das, A. K.; Aich, K.; Nandi, P. K. Chem. Commun. 2013, 49, 2912.

[25] Liang, C.; Jiang, S. Analyst 2017, 142, 4825.

[26] Geng, L.; Yang, X. F.; Zhong, Y.; Li, Z.; Li, H. Dyes Pigm. 2015. $12,213$.

[27] Zhang, H.; Huang, Z.; Feng, G. Anal. Chim. Acta 2016, 920, 72.

[28] Goswami, S.; Manna, A.; Mondal, M.; Sarkar, D. RSC Adv. 2014, 4, 62639 .
[29] Huang, Q.; Yang, X. F.; Li, H. Dyes Pigm. 2013, 99, 871.

[30] Liu, Y.; Feng, G. A. Org. Biomol. Chem. 2014, 12, 438.

[31] Huang, C.; Jia, T.; Yu, C.; Zhang, A.; Jia, N. Biosens. Bioelectron. 2015, 63, 513 .

[32] Svechkarev, D.; Dereka, B.; Doroshenko, A. J. Phys. Chem. A 2011, $115,4223$.

[33] Santra, M.; Roy, B.; Ahn, K. H. Org. Lett. 2011, 13, 3422.

[34] Chang, I. J.; Hwang, K. S.; Chang, S. K. Dyes Pigm. 2017, 137, 69

[35] Zhao, J.; Zhao, Y.; Xu, S.; Luo, N.; Tang, R. Inorg. Chim. Acta 2015, 438, 105.

[36] Yang, C.; Chen, Y.; Wu, K.; Wei, T.; Wang, J.; Zhang, S.; Han, Y. Anal. Methods 2015, 7, 3327.

[37] Liu, B.; Wang, H.; Wang, T.; Bao, Y.; Du, F.; Tian, J.; Li, Q.; Bai, R. Chem. Commun. 2012, 48, 2867.

[38] Cui, L.; Zhu, W.; Xu, Y.; Qian, X. Anal. Chim. Acta 2013, 786, 139.

[39] Luo, W.; Li, J.; Liu, W. Org. Biomol. Chem. 2017. 15. 5846.

[40] Tuo, M.; Jin, Z.; Liu, L.; Yun, H.; Zhang, Z. Chin. J. Org. Chem. 2014, 34, 1780 (in Chinese) (马拓, 张瑾, 刘龙珠, 贺云, 张尊听, 有机化学, 2014, 34, 1780.)

[41] Gupta, V. K.; Mergu, N.; Singh, A. K. Sens. Actuators, B 2014, 202, 674.

[42] Murale, D. P.; Kim, H.; Choi, W. S.; Churchill, D. G. Org. Lett. 2013, 15, 3946.

[43] Li, G.; Zhu, D.; Liu, Q.; Xue, L.; Jiang, H. Org. Lett. 2013, 15, 924

[44] Wang, L.; Zang, Q.; Chen, W.; Hao, Y.; Liu, Y. N.; Li, J. RSC Adv. 2013, 3, 8674 .

[45] Liu, B.; Wang, J.; Zhang, G.; Bai, R.; Pang, Y. ACS Appl. Mater. Interfaces 2014, 6, 4402.

[46] Liu, Y.; Yu, D.; Ding, S.; Xiao, Q.; Guo, J.; Feng, G. ACS Appl. Mater. Interfaces 2014, 6, 17543.

[47] Zhang, Y.; Wang, J.-H.; Zheng, W.; Chen, T.; Tong, Q.-X.; Li, D. J. Mater. Chem. B 2014, 2, 4159 .

[48] Goswami, S.; Manna, A.; Paul, S.; Das, A. K.; Nandi, P. K.; Maity, A. K.; Saha, P. Tetrahedron Lett. 2014, 55, 490.

[49] Kim, T. I.; Kang, H. J.; Han, G.; Chung, S. J.; Kim, Y. Chem. Commun. 2009, 5895.

[50] Fan, C.; Luo, S.; Qi, H. Luminescence 2016, 31, 423.

[51] Hu, Q.; Zeng, F.; Yu, C.; Wu, S. Sens. Actuators, B 2015, 220, 720.

[52] Gao, M.; Hu, Q.; Feng, G.; Tang, B.-Z.; Liu, B. J. Mater. Chem. B 2014, 2, 3438 .

[53] Jin, X.; Sun, X.; Di, X.; Zhang, X.; Huang, H.; Liu, J.; Ji, P.; Zhu, H. Sens. Actuators, B 2016, 224, 209.

[54] Goswami, S.; Das, S.; Aich, K.; Pakhira, B.; Panja, S.; Mukherjee, S. K.; Sarkar, S. Org. Lett. 2013, 15, 5412.

[55] Jin, X.; Liu, C.; Wang, X., Huang, H.; Zhang, X.; Zhu, H. Sens. Actuators, B 2015, 216, 141 .

[56] Liu, B.; Liu, Q.; Shah, M.; Wang, J.; Zhang, G.; Pang, Y. Sens. Actuators, B 2014, 202, 194.

[57] Ju, Z.; Shu, P.; Xie, Z.; Jiang, Y.; Tao, W. Chin. J. Org. Chem. 2019, 39, 697 (in Chinese).

(鞠志宇，舒朋华，谢智宇，蒋雨晴，陶伟杰，许志红，有机化学， 2019, 39, 697.) 